Int. J. Speleol. 10 (1978), pp. 195 - 203

\title{
Observations sur les comportements de Lucifuga Subterranea Poey (Pisces, Ophidiidae), poisson cavernicole de Cuba
}

\author{
par \\ G. THINÈS et M. PIQUEMAL \\ «Observations on the behaviour patterns of the cuban cave fish Lucifuga subterranea Poey \\ (Pisces, Ophidiidae)»
}

\begin{abstract}
SUMMARY
The swimming behaviour and the sensory reactions of the blind cuban cave fish Lucifuga subterranea Poey were studied on a single individual during a period of 6 months. Mechanical stimulation elicits but slight reactions. Gustatory substances in solution elicit fairly typical motor responses which are not followed by systematic exploration behaviour, the same being true for stimulations by odours of prey. The presence of moving prey provokes an oriented exploration with a slight plunging movement of the kind evidenced in other cave fishes. Actual seizing of the prey requires an active approach of the latter towards the anterior part of the body of the fish.
\end{abstract}

Plusieurs missions du C.N.R.S. effectuées en 1974 et en 1975 avaient permis de récolter un matériel zoologique important dont de nombreaux exemplaires fixés du Poisson cavernicole Lucifuga subterranea. Des spécimens vivants de la même espèce qui avaient été rapportés en France à la même occasion se révélèrent fragiles et ne survécurent que pendant quelques semaines dans l'installation pourtant fort bien aménagée du Laboratoire Souterrain du C.N.R.S. à Moulis. Un seul individu ayant survécu pendant deux ans environ, il fut décidé de procéder sur celui-ci à diverses observations. Bien que notre étude se limite à un seul animal, nous estimons que la rareté relative des undividus vivants disponibles en laboratoire nous obligeait à mettre à profit au maximum la possibilité assez exceptionnelle d'observer les comportements d'un Ophidiidae cavernicole en aquarium. L'individu dont nous disposions avait une longueur totale de $105 \mathrm{~mm}$.

* Laboratoire Souterrain du C.N.R.S. Moulis (Ariège)

et Centre de psychologie experimentale et comparée, Université de Louvain, B - 3041 Pellenberg (Belgique) 


\section{LA STRUCTURE DE LA LOCOMOTION.}

La nage de l'individu que nous avons observé est extrêmement lente. En dehors de toute perturbation, la vitesse de l'animal, mesurée en plaçant une règle transparente le long de l'aquarium et en chronométrant les distances couvertes par le poisson, est de $10 \mathrm{~mm} / \mathrm{sec}$ environ. Comme l'avait déjà noté Eigenmann (1909), le Lucifuga nage dans des positions très variables, la face dorsale vers le haut ou orientée latéralement. En outre, le poisson nage à reculons. La nage à reculons s'effectue souvent dans des passages étroits. Elle rappelle le type de nage à reculons que l'on observe chez les poissons électriques de la famille des Mormyridae. La position tête vers le bas est fréquente.

Quant aux mouvements des nageoires, ils constituent une structure motrice intégrée complexe dans laquelle on peut distinguer:

1) Une onde continue de la dorsale se propageant de l'avant vers l'arrière.

2) Une onde continue parallèle et synchrone de l'anale se propageant également de l'avant vers l'arrière.

3) Un mouvements latéral du petit pédoncule caudal.

4) Des mouvements des pectorales. Ceux-ci, comme le remarque Eigenmann (1909) ne sont pas synchronisés et interviennent de façon indépendante.

Les mouvements synchronisés de la dorsale et de l'anale sont indissociables dans la locomotion et sont responsables de l'impulsion principale qui imprime au corps son mouvement natatoire dans le déplacement ordinaire à vitesse lente. Les accélérations brusques du corps sont dues à un mouvement de torsion du tronc, lequel n'intervient pas dans le déplacement lent. Il est à noter que dans la locomotion lente ordinaire, le pédoncule caudal exécute un faible mouvement latéral chaque fois qu'une onde synchrone de la dorsale et de l'anale atteint l'extrémité de ces deux nageoires.

Les ventrales, réduites à deux courts filaments ne semblent intervenir ni dans la propulsion, ni dans l'équilibration.

Le poisson exécute également un mouvement courbe du tronc dans lequel la partie postérieure du corps est ramenée vers la tête. Ce mouvement est exceptionnel et semble lié à l'exploration du substrat; il est d'autant plus marqué que le corps est plus incliné vers le bas. Il disparaît toutefois dès que l'animal adopte une position verticale, le museau étant à proximité du substrat.

\section{L'EXPLORATION DU MILIEU.}

Les observations ont été effectuées dans un grand aquarium de 70×30×40 cm dans lequel la hauteur de l'eau était de $30 \mathrm{~cm}$. Le volume total d'eau disponible était donc de 63 litres.

Les modalités de l'exploration du Lucifuga sont très différentes de celles qui ont été relevées chez d'autres poissons cavernicoles comme l'Anoptichthys 
gen.(1) et le Caecobarbus geertsi, formes qui présentent une activité continue et relativement élevée (Thinès, Soffié et Vandenbussche, 1966 - Thinès et Wissocq, 1972 - Heuts et Thinès, 1971). Contrairement aux deux formes précitées, le Lucifuga est faiblement actif et se déplace très lentement, le corps généralement orienté vers le haut à $\pm 45^{\circ}$.

Dans le volume d'eau de l'aquarium expérimental utilisé, le poisson manifeste une préférence marquée pour la partie inférieure proche du substrat. La hauteur de l'eau ayant été divisée en 3 zones égales de $10 \mathrm{~cm}$ au moyen de 2 repères colorés, 5 observations indépendantes de 15 minutes furent effectuées. Au cours de ce temps total de 75 minutes, le poisson ne quitta la zone inférieure que pendant $86 \mathrm{sec}$. Ce résultat contraste nettement avec ce que l'on observe chez l'Anoptichthys. Ce dernier explore activement tout le volume d'eau disponible et ne plonge vers le substrat qu'à la suite d'une stimulation chimique ou mécanique. La préférence du Lucifuga pour la zone inférieure du milieu est plus continue et ne dépend pas exclusivement d'une stimulation spécifique.

\section{RÉPONSES A DES STIMULATIONS SENSORIELLES SPÉCIFIQUES.}

Nous avons observé les réactions du Lucifuga aux principales stimulations sensorielles dont la signification biologique est théoriquement la plus évidente dans le milieu souterrain, c.à.d. les stimulations mécaniques et les stimulations chimiques. Il y aurait lieu d'y ajouter les réponses aux modifications thermiques, mais celles-ci exigent d'être étudiées sur un matériel numériquement abondant si l'on entend constituer des groupes expérimentaux indépendants. Nos observations ont été étalées de façon irrégulière sur une periode d'environ 6 mois. Les deux observateurs qui effectuèrent les expériences avaient travaillé initialement ensemble; ils effectuèrent ultérieurement des observations indépendantes qui se révélèrent convergentes sur le plan descriptif et qui permirent de considérer que l'ensemble des comportementes décrits constituait un tout suffisamment homogène. Toutes les observations furent effectuées à la température de $24^{\circ} \mathrm{C}$ dans un local faiblement éclairé.

\section{a) Réponses à la stimulation mécanique.}

La stimulation mécanique pratiquée en imprimant un choc bref sur la paroi de l'aquarium au moyen du doigt ou d'un petit marteau en caoutchouc détermine chez les poissons cavernicoles que nous avions étudiés antérieurement des réactions assez nettes, en particulier chez l'Anopthichthys, qui dans ces condi-

(1) Le genre Anoptichthys n'etant plus admis, l'ancienne dénomination Anoptichthys jordani doit être remplacée par Astyanax jordani pour qualifier la forme cavernicole de l'Astyanax mexicanus (cf. Sadoglu, 1957). 
tions s'immobilise brusquement puis plonge vers le substrat (Thinès, Soffié et Vandenbussche, 1966), la même réaction en deux temps s'observant avec une moindre intensité chez le Caecobarbus (Thinès et Wissocq, 1972). Une telle stimulation n'entraîne pratiquement aucune réponse chez Typhlogarra widdowsoni, lequel ne manifeste sous l'effet d'un choc mécanique qu'un bref écartement de toutes les nageoires tout en restant sur place (Marshall et Thinès, 1958). Chez le Lucifuga, la stimulation mécanique des parois de l'aquarium exerce un effet indubitable mais peu reproductible. Dans les cas où l'animal réagit, il a tendance à incliner le corps en direction de la source de stimulation et à accélérer très légèrement sa nage. Des chocs répétés à des intervalles de $30 \mathrm{sec}$ ou de 1 minute n'ont plus d'effet à partir de la deuxième stimulation. Lorsque la stimulation est peu intense, le poisson incline généralement le corps vers le'bas à 45 degrés et amorce parfois des mouvements de nage très lents en maintenant cette orientation du corps. La réaction peut être observée lors de certaines stimulations et être absente à la suite de stimulations identiques données après un intervalle de temps très long ( 30 minutes et plus). Néanmoins, la structure motrice qui réapparaît chaque fois que la stimulation est efficace est caractérisée par une inclinaison du corps vers le bas et un maintien prolongé de celle-ci avant que la nage lente s'amorce. Aucune exploration active du substrat, du genre de celle qui est typique de l'Anoptichthys, n'est jamais observée. Ce type de réponse peut être décrit qualitativement comme une structure réactionnelle intermédiaire entre les reponses vives de l'Anoptichthys et relativement vives du Caecobarbus d'une part, et les réponses faibles, dénuées de composante locomotrice, du Typhlogarra, d'autre part.

\section{b) Réponses à la stimulation chimique de substances gustatives en solution.}

Dans tous ces essais, la solution ètait versée dans l'aquarium lorsque le poisson était à une distance de 5 à $10 \mathrm{~cm}$ de la surface. L'introduction dans l'aquarium à l'aplomb de l'animal de 3 gouttes d'une solution de $\mathrm{NaCl}$ à $5 \%$ provoque un léger mouvement d'exploration après 10 à 12 secondes dans les cas les plus favorables ( 5 essais espacés). La même stimulation répétée 3 minutes plus tard n'entraîne aucune réaction.

L'introduction dans l'aquarium de 3 gouttes d'une solution d'acide acétique à $1 \%$ produit le même effet mais le temps de réaction est plus long (de 16 à 45 sec). Dans un cas, l'animal a plongé verticalement vers le substrat pendant 3 à 4 secondes et a effectué des mouvements buccaux violents et saccadés. Sur un total de 9 essais espacés, le poisson n'a réagi à la solution d'acide acétique que dans 3 cas. La stimulation au moyen de 3 gouttes d'une solution de quinine à $0,5 \%$ ne provoque une réponse que dans 2 essais sur un total de 9 essais espacés. Celle-ci se traduit par un léger redressement au bout de 10 secondes, suivi 
d'une descente lente vers le substrat sans exploration. Sur 5 essais espacés, l'introduction de 3 gouttes d'une solution de glucose à $25 \%$ ne provoque une réponse que dans 2 cas, celle-ci se traduisant par une nage brève après 0,5 seconde. Dans l'ensemble, les réponses sont faibles et irrégulières et ne peuvent être comparées aux réactions très nettes qui furent observées par Humbach (1960) sur Anoptichthys à des solutions correspondant aux quatre qualités gustatives fondamentales. 8 essais espacés de contrôle effectués chaque fois avec 3 gouttes d'eau distillée n'entraînent une réponse que dans 2 cas. Ces résultats indiquent donc en première approximation que la présence de stimulus salés, sucrés, amers et acides est perçue par le Lucifuga.

Les réponses sont toutefois peu différenciées et il semble exclu de songer à utiliser les faibles réactions motrices notées dans un schéma de dressage permettant de déterminer le seuil absolu des quatre qualités gustatives. Ces indications devraient bien entendu être vérifiées sur des groupes numériquement suffisants.

\section{c) Réponses à l'odeur de proies.}

Les stimulus utilisés dans ces expériences étaient des broyats d'Artemia et de larves de Chironomus. Les proies étaient broyées (3 Artemia ou 3 larves de Chironomus selon le cas) dans $2 \mathrm{cc}$ d'eau distillée que l'on filtrait. Le filtrat était utilisé comme stimulus chimique à raison de 3 gouttes par essai comme dans les expériences utilisant des solutions.

\section{Réponses à l'odeur d'Artemia salina.}

8 essais espacés au total ont été effectués en utilisant l'odeur d'Artemia comme stimulus. Dans ces expériences, l'animal était affamé depuis 3 à 5 jours et les stimulus étaient introduits dans l'aquarium lorsque le poisson était à 2 ou 3 $\mathrm{cm}$ de la surface. Une réponse positive fut observée dans 6 de ces 8 essais après un temps assez variable allant de 3 à 90 secondes. Dans les cas où le poisson réagit, la réponse se traduit par un arrêt brusque de la nage lente suivi d'une période d'inaction variable elle aussi et allant de 2 à 30 secondes, après quoi le poisson se remet à nager lentement vers le haut ou vers le bas. Aucune exploration active ne fait suite à ces réactions sensorielles.

\section{Réponses à l'odeur de larves de Chironomus.}

7 essais espacés au total ont été effectués en utilisant l'odeur de larves de $C h i$ ronomus comme stimulus. Dans ces expériences, l'animal n'avait pas été affamé et les stimulus étaient introduits dans l'aquarium lorsque le poisson était à 4 ou $10 \mathrm{~cm}$ de la surface. Une seule réponse positive fut observée au cours de ces 7 essais 2 secondes après l'introduction du stimulus. Ici encore, on observe 
un arrêt brusque de la nage lente suivi d'une période d'inaction (durée: 12 secondes), après quoi l'animal se remet à nager et gagne le fond où il procède à une exploration peu active, tête en bas, avec de légers mouvements de la caudale repliée.

d) Réponses à l'odeur d'une proie couplée à la présence de la même proie vivante.

Une unique séance d'observation fut effectué dans laquelle 5 larves vivantes de Chironomus furent introduites dans l'aquarium après que le poisson eût réagi positivement à l'odeur du filtrat, préparé et introduit dans l'eau selon le procédé antérieurement adopté. Lors de l'introduction du filtrat, le poisson était à $1 \mathrm{~cm}$ de la surface et il réagit après 1 seconde par une nage à reculons, une plongée vers le fond et une exploration consécutive peu active. On introduit à ce moment dans l'aquarium 5 larves de Chironomus vivantes que le poisson suit lentement et happe, l'exploration et la saisie prenant 2 minutes. Il nage alors à reculons, parcourt lentement l'aquarium et revient vers les 4 larves restantes, explorant le fond tête vers le bas. Une seconde larve est happée 330 secondes après la première. Une heure plus tard, le poisson est toujours à l'aplomb des larves. Une troisième larve est consommée 9 heures plus tard. Cette observation prolongée montre que contrairement à l'odeur seule, la présence effective d'une proie vivante déclenche une exploration orientée suivie de prédation. Les observations qui vont suivre confirment cette constatation.

\section{e) Réponses à la présence de proies vivantes.}

9 séances d'observation étalées sur 13 semaines furent consacrées à l'ètude du comportement de prédation du Lucifuga en présence d'Artemia vivantes et 3 séances d'observation étalées sur 4 semaines furent consacrées au comportement de prédation du poisson en présence de larves vivantes de Chironomus.

\section{1) Prédation en présence d'Artemia salina.}

Au cours des 3 premières séances, 4 individus d'Artemia furent introduits dans l'aquarium le poisson étant sur le fond. La première séance est négative. Au cours de la deuxième séance, une Artemia nageant à proximité du poisson est happée 4 minutes après avoir été introduite dans l'eau. Le Poisson s'immobilise 0,5 seconde avant de la happer. La prédation est suivie d'une nage accélérée au cours de laquelle le Lucifuga explore activement le fond, tête en bas, avec de légers mouvements de la caudale repliée. La structure de l'exploration est la même que celle observée en C - 2 pour l'odeur seule de larves de Chironomus. La même séance répétée 4 jours plus tard fournit un résultat identi- 


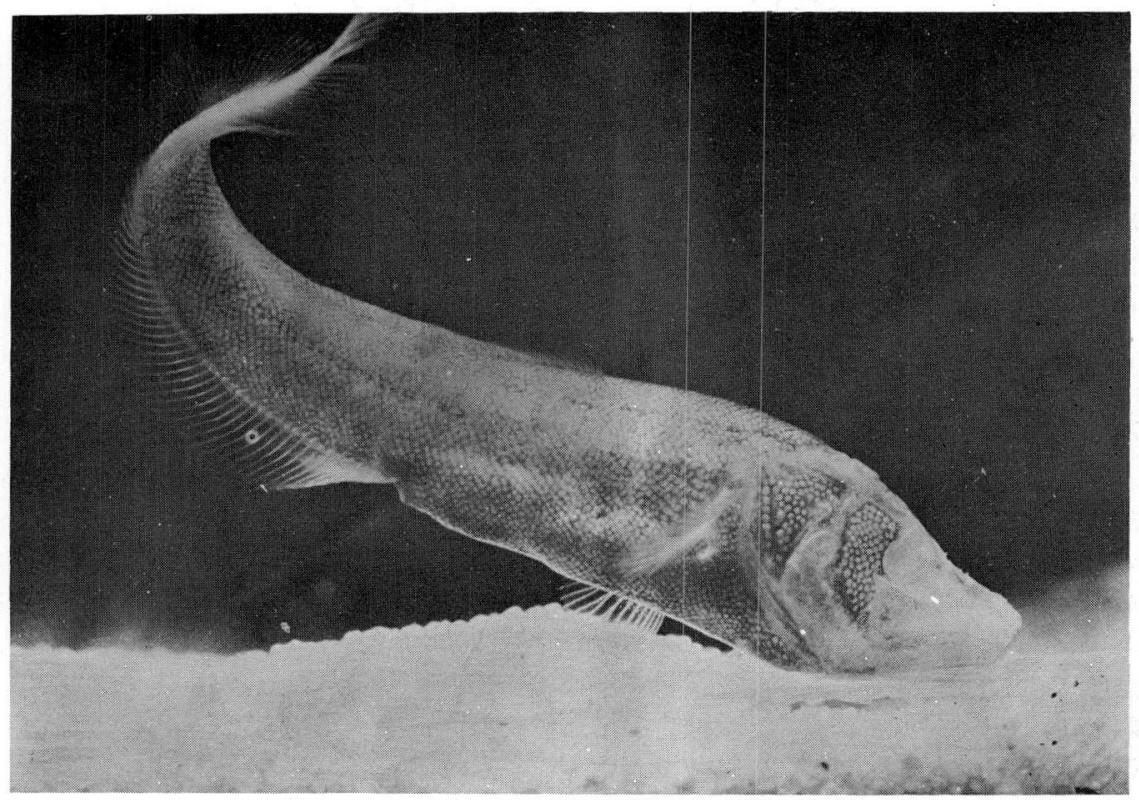

Lucifuga subterranea, poisson cavernicole de Cuba.

(Photo Laboratoire Souterrain du C.N.R.S. à Moulis, France)

que. Au cours des 4 séances suivantes, 5 individus d'Artemia furent utilisés et introduits dans l'eau, ici encore quand le poisson était sur le fond. Dans tous les cas, l'exploration est active. L'ensemble du processus peut être analysé comme suit:

- Dès que la stimulation due à l'odeur et aux mouvements des Artemia atteint le poisson, celui-ci remonte vers la surface en direction du point de distribution de la nourriture. Cette réaction initiale intervient 1 seconde à 1 minute après l'introduction des proies dans l'eau.

- Cette première réponse est suivie d'une période d'immobilité d'une durée de 1 à 6 minutes.

- Si le poisson est éloigné des proies, il explore en adoptant la position tête vers le bas et caudale repliée comme en C-2 et en E-1. Cette exploration peut l'amener à proximité des proies sans qu'il parvienne à les saisir. Dans ce cas, il continue son exploration pendant un temps plolongé.

- Si au contraire le Poisson est à proximité des proies du fait que celles-ci se sont rapprochées activement de lui, il poursuit celles-ci et les happe. Ce comportement de prédation n'est cependant efficace que si la proie s'est trouvée en mouvement à proximité du museau pendant 4 à 5 secondes. Après la saisie, le Lucifuga nage généralement à reculons.

- Après une exploration infructeuse prolongée, le poisson a tendance à revenir 
vers la surface à proximité du point de distribution de la nourriture.

Les deux dernières séances, dans lesquelles 3 individus d'Artemia furent utilisés comme proies, confirment les observations effectuées au cours des 7 séances précédentes.

\section{z) Prédation en présence de larves de Chironomus.}

Une séance dans laquelle ou utilisait 3 larves comme proies et deux séances dans lesquelles on en utilisait 4 permettent de mettre en évidence la même structure d'exploration. Aucune saisie directe n'est observée, mais quelques larves sont happées sur le fond après un temps prolongé. Il semble que les mouvements des larves de Chironomus soient moins bien détectés que ceux des Artemia et que la prédation des larves survienne surtout sur le fond après une exploration prolongée pouvant s'étaler sur plusieurs heures et entrecoupée de périodes d'immobilité. Ces dernières observations confirment les faits rapportés au point $\mathrm{D}$.

\section{DISCUSSION ET CONCLUSIONS}

L'examen descriptif des réactions sensorielles de Lucifuga subterranea permet de tirer quelques conclusions d'ensemble en lien avec le type natatoire très particulier de cette espèce.

1. Tant dans le cas de la stimulation mécanique que dans celui de la stimulation chimique par des substances en solution, le premier signe de réponse est une inclinaison du corps suivie d'une nage lente vers le fond. L'orientation vers le substrat n'a toutefois nullement la netteté de la réponse plongeante rapide de l'Anoptichthys dans ces conditions, telle qu'elle est décrite par Thinès, Soffié et Vandenbussche (1966).

2. Tant dans le cas de la stimulation par des odeurs de proies que dans celui de la stimulation par proies vivantes mobiles, la structure réactionnelle typique comporte une phase initiale d'immobilisation suivie d'une nage lente orientée,ici encore, vers le substrat. Toutefois, l'exploration active ne s'observe qu'en présence de proies vivantes.

3. La capture de proies vivantes ne s'opère avec efficacité et dans un délai relativement bref que si les proies se dirigent activement vers le poisson et passent à proximité de son museau. En d'autres termes, l'interaction prédateur-proie est principalement réglée par l'activité de la proie et c'est cette dernière qui déclenche la saisie précise.

4. La découverte de proies passives sur le fond est longue et imprécise; elle n'aboutit que dans un petit nombre de cas et exige une au plusieurs périodes d'exploration prolongée. Ce fait est d'ailleurs général chez les poissons cavernicoles, même chez l'Anoptichthys qui, tout en étant très actif, localise sa 
proie avec difficulté encore qu'en un temps beaucoup plus court, allant de une à plusieurs minutes.

5. Le Lucifuga présente dans l'ensemble un comportement alimentaire dont la structure est typiquement celle des espèces étudiées à ce jour, telles que l'Anoptichthys et le Caecobarbus en particulier. Chez le Lucifuga comme chez ces autres espèces, l'exploration consécutive à la stimulation chimique amène souvent l'animal à proximité immédiate de la proie sans qu'aucune tentative active de saisie s'ensuive. C'est là, semble-t-il, un trait propre des poissons cavernicoles qui ne s'explique pas entièrement par une absence de motivation alimentaire, vu que ce comportement survient souvent après une réponse sensorielle intense et fasse suite à la cessation brusque d'une exploration nettement orientée vers la proie quelques secondes plus tôt.

6. Nos observations en aquarium ne nous ont pas permis d'observer le comportement de fuite orienté avec précision vers des anfractuosités qui est rapporté par Eigenman (1909) comme une conduite caractéristique en milieu naturel, tant chez le Lucifuga que chez le Stygicola. Cette conduite, qui témoigne de l'existence d'une bonne mémoire topographique était déclenchée par une perturbation mécanique. Nous n'avons rien observé de semblable lorsque nous pratiquions ce type de stimulation. Par contre, l'inclinaison typique du corps au cours de la nage libre notée par le même auteur, est confirmée dans nos conditions d'observation.

Les auteurs remercient M. le Professeur Cl. Delamare Deboutteville, Directeur du Laboratoire Souterrain du C.N.R.S. à Moulis qui a aimablement mis à leur disposition le matériel zoologique. Ils remercient également M.C. Juberthie, sous-directeur du Laboratoire Souterrain et M.J. Durand qui leur ont fourni une aide appréciable dans la réalisation des observations.

\section{BIBLIOGRAPHIE.}

EIGENMANN C., 1909 - Cave Vertebrates of America. A study in degenerative evolution. Carn. Inst. Wash. Publ. $\mathrm{n}^{\circ} 104$.

HEUTS B. and THINĖS G., 1971 - Behavioural changes in a stock of Barbus conchonius Hamilton (Pisces, Cyprinidae) after accidentally induced partial depigmentation and blindness. Z. Tierspychol. $28: 113-163$.

HUMBACH I., 1960 - Geruch und Geschmack bei den augenlosen Hölenfischen Anoptichthys jordani Hubbs une Innes und Anoptichthys hubbsi Alvarez. Diss. Univ. Köln et Naturwiss, 23 : 551 .

MARSHALL N.B. and THINÈS G., 1958 - Studies of the brain, sense organs and light sensitivity of a blind cave fish Typhlogarra widdowsoni from Iraq. Proc. Zool. Soc. London, 3 : 441-456. SADOGLU P., 1957 - Mendelian inheritance in the hybrids between the Mexican blind cave fishes and their overground ancestor. Verh. Dtsch. Zool. Ges. Graz, 432-439.

THINÈS G., SOFFIÉ M. et VANDENBUSSCHE E., 1966 - Analyse du comportement alimentaire du poisson cavernicole aveugle Anoptichthys Gen. et d'hybrides F 1 Astyanax Anoptichthys et F2. Intern. J. Speleol. 2 : 437-448.

THINÈS G., et WISSOCQ N., 1972 - Etude comparée du comportement alimentaire de deux poissons cavernicoles Anoptichthys jordani hubbs et Innes et Caecobarbus geertsi Blgr. Intern. J. Speleol., $4: 139-169$. 